\title{
Comment on "The timing of parathyroid hormone measurement defines the cut-off values to accurately predict postoperative hypocalcemia: a prospective study"
}

\author{
Adriana Josefina Ortiz-Morales ${ }^{1} \cdot$ Carolina Castillo-Castro $^{1} \cdot$ Leonardo G. Mancillas-Adame $^{1}$
}

Received: 13 July 2018 / Accepted: 13 August 2018 / Published online: 18 August 2018

(c) Springer Science+Business Media, LLC, part of Springer Nature 2018

To the Editor,

We read with interest the article by Filho et al. [1], in which the investigators aimed to evaluate the possible association between the timing of iPTH measurement and post-operative hypocalcemia. The authors concluded that patients with iPTH above $19.55 \mathrm{pg} / \mathrm{ml} 4 \mathrm{~h}$ after surgery, or $14.35 \mathrm{pg} / \mathrm{ml}$ on the first morning after surgery can be safely discharged from hospital without supplemental calcium and/or vitamin D and while the conclusions are profoundly interesting, and iPTH measurement value as a predictor for hypoparathyroidism is recognized, its use has not gained enough traction.

Regarding the validity of the study assumptions we are concerned about the lack of a strict protocol on the timing of post-surgical iPTH sampling; almost one-third of the patients were tested in the first $2 \mathrm{~h}, 11.8 \%$ between 2 and $3 \mathrm{~h}$ post thyroidectomy, and only the remaining $61.4 \%(n=62)$ were sampled for measurement of iPTH at $4 \mathrm{~h}$. Various studies [2,3] have examined the utility of measuring intraoperative or postoperative intact PTH levels drawn at various time points in the post-thyroidectomy period, and the timing of PTH measurements in published studies has ranged from $10 \mathrm{~min}$ to $24 \mathrm{~h}$ post-thyroidectomy. Still, there is a significant variation in thresholds to predict hypocalcemia in the published literature. Consequently, based on the dissimilar moments the peripheral venous blood was collected, we consider that it is difficult to support the authors' conclusion on the 4-h cut-off iPTH value, and might not be appropriate to consider it as a standardized cut-

Leonardo G. Mancillas-Adame

leonardo.mancillasd@uanl.mx

1 Endocrinology Division, Department of Internal Medicine, University Hospital "Dr. Jose E. Gonzalez", Universidad Autonoma de Nuevo León, Monterrey, Mexico off value to predict hypocalcemia. A sensitivity analysis using only the $60 \%$ of the subjects who were actually measured at $4 \mathrm{~h}$, might provide some reassurance.

Still, we consider this proposal interesting, since these findings might have important implications for clinical practice. The conclusion from this study would need to be tested in prospective trials following a strict protocol, and if proven and replicated, it would be of relevance in our everyday practice.

\section{Compliance with ethical standards}

Conflict of interest The authors declare that they have no conflict of interest.

\section{References}

1. E.B.Y. Filho, R.V. Machry, R. Mesquita, R.S. Scheffel, A.L. Maia, The timing of parathyroid hormone measurement defines the cutoff values to accurately predict postoperative hypocalcemia: a prospective study. Endocrine 61(2), 224-231 (2018). https://doi. org/10.1007/s12020-018-1601-9

2. Z. Sahli, A. Najafian, S. Kahan, E.B. Schneider, M.A. Zeiger, A. Mathur, One-hour postoperative parathyroid hormone levels do not reliably predict hypocalcemia after thyroidectomy. World J. Surg. 42(7), 2128-2133 (2018). https://doi.org/10.1007/s00268-0174444-2

3. J.S. Jumaily, J.P. Noordzij, A.G. Dukas, S.L. Lee, V.J. Bernet, R.J. Payne, I.K. McLeod, M.P. Hier, M.J. Black, P.D. Kerr, M. Raffaelli, R. Bellantone, C.P. Lombardi, M.S. Dietrich, Prediction of hypocalcemia after using 1- to 6-hour postoperative parathyroid hormone and calcium levels: an analysis of pooled individual patient data from 3 observational studies. Head Neck 32(4), 427-434 (2010). https://doi.org/10.1002/hed.21199 